\title{
Transitionless Quantum Driving in Spin Echo
}

\author{
Anton Gregefalk and Erik Sjöqvist@* \\ Department of Physics and Astronomy, Uppsala University, Box 516, SE-751 20 Uppsala, Sweden
}

(Received 13 July 2021; revised 3 December 2021; accepted 21 January 2022; published 3 February 2022)

\begin{abstract}
Spin echo can be used to refocus random dynamical phases caused by inhomogeneities in control fields and thereby retain the purity of a spatial distribution of quantum spins. This technique for accurate spin control is an essential ingredient in many applications, such as nuclear magnetic resonance, magnetic resonance imaging, and quantum information processing. Here, we show how all the elements of a spin echo sequence can be performed at high speed by means of transitionless quantum driving. This technique promises accurate control of rapid quantum spin evolution. We apply the scheme to universal nonadiabatic geometric single- and two-qubit gates in a nuclear magnetic resonance setting.
\end{abstract}

DOI: 10.1103/PhysRevApplied.17.024012

\section{INTRODUCTION}

Techniques for accurate spin control are an essential ingredient in physics and chemistry. One such technique is spin echo [1,2], in which a spin is taken along a path twice, where the second path exactly retraces the first but in the opposite direction, and where the paths are surrounded by short $\pi$ pulses. In this way, it becomes possible to refocus random dynamical phases caused by spatial inhomogeneities in the control fields and thereby retain the purity of the spin ensemble.

More generally, the sensitive nature of the quantum regime makes the system prone to errors, where tiny disturbances can make the quantum nature of the system disappear. This becomes especially challenging in quantum information processing (QIP), as loss of coherence removes the advantages of using quantum degrees of freedom as information carriers. Therefore, various refocusing techniques have been used in QIP, such as, in the implementation of robust geometric gates in adiabatic cyclic evolution, in which the use of Berry phases [3] removes the dependence on experimental details $[4,5]$.

The use of adiabatic evolution in spin echo prolongs the exposure to errors of quantum-mechanical origin, such as environment-induced decoherence and decay. This calls for a decrease in the run time of the adiabatic paths. Here, we address this problem by combining the idea of spin echo with transitionless quantum driving (TQD) [6] to

*erik.sjoqvist@physics.uu.se

Published by the American Physical Society under the terms of the Creative Commons Attribution 4.0 International license. Further distribution of this work must maintain attribution to the author(s) and the published article's title, journal citation, and DOI. Funded by Bibsam. shorten the run time and to suppress nonadiabatic transitions. This scheme opens up the possibility of precise control of high-speed evolution of quantum spins, which may help to improve the accuracy of various technological applications, such as nuclear magnetic resonance (NMR) [7], magnetic resonance imaging (MRI) [8], and QIP [9].

\section{THE SCHEME}

We consider a spin echo scheme based on closed loops of adiabatic control parameters. Our basic physical setting is a spin qubit in an NMR system in which a radio frequency (rf) magnetic field in the $x y$ plane is added to a bias magnetic field in the $z$ direction. The latter is detuned in a rotating frame by the frequency of the rf field. By sweeping the $\mathrm{rf}$ frequency not all away to resonance, the initial spin can be made to point at an arbitrary angle $\theta$ to the rotational $z$ axis. The spin is thereafter taken around a cone-shaped loop by rotating the rf field slowly with angular frequency $\omega$ around the $z$ axis $[4,5]$. This implies that the two orthogonal spin states (we consider spin $s=1 / 2$ ) pick up Berry phases $\beta_{ \pm}=\mp \pi(1-\cos \theta)$. A second loop surrounded by short $\pi$ pulses exactly retraces the first loop but in the opposite direction. The basic adiabatic sequence is shown in Fig. 1.

We now demonstrate how TQD can be used to implement a modified scheme, in which all components of the spin echo sequence can be performed at high speed. While our approach is adapted to NMR, a similar technique has been used to measure the Berry phase at high speed in a superconducting phase qubit [10].

Consider a spin- $1 / 2$ particle in a time-dependent magnetic field $\mathbf{B}_{0}=B_{0}(\sin \theta \cos \omega t, \sin \theta \sin \omega t, \cos \theta)$, defining the "root" Hamiltonian

$$
H_{0}(t)=\gamma \mathbf{B}_{0}(t) \cdot \mathbf{S}
$$


(a)

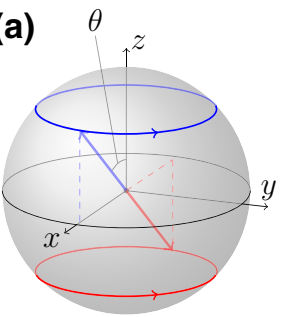

(c) $\theta$

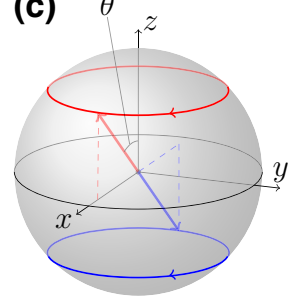

(b)

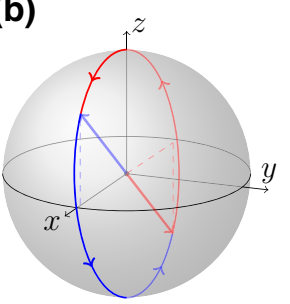

(d)

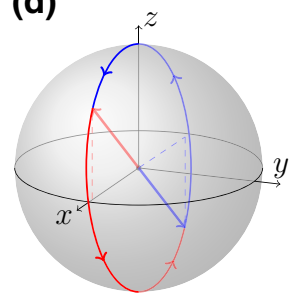

FIG. 1. The basic spin echo sequence. (a),(c) are the two closed adiabatic spin loops traced in opposite directions. (b),(d) are short $\pi$ transformations, half-circle rotations of the spin around the $y$ axis.

of the TQD setting. Here, $\gamma$ is the gyromagnetic ratio and $\mathbf{S}=(1 / 2) \hbar \boldsymbol{\sigma}$ with $\boldsymbol{\sigma}=\left(\sigma_{x}, \sigma_{y}, \sigma_{z}\right)$ the standard Pauli operators. Nonadiabatic transitions between the instantaneous eigenstates $\left|\phi_{ \pm}(t)\right\rangle$ of $H_{0}(t)$ are completely suppressed by adding a correction term, yielding the TQD Hamiltonian [6]

$$
H(t)=\left[\gamma \mathbf{B}_{0}(t)+\mathbf{b}_{0}(t) \times \partial_{t} \mathbf{b}_{0}(t)\right] \cdot \mathbf{S}=\gamma \mathbf{B}(t) \cdot \mathbf{S}
$$

with $\mathbf{b}_{0}(t)=\mathbf{B}_{0}(t) /\left|\mathbf{B}_{0}(t)\right|$ the direction of the magnetic field. In this way, the spin prepared at $t=0$ in an eigenstate $\left|\phi_{ \pm}(0)\right\rangle$ of $H_{0}(0)$ exactly monitors the instantaneous eigenstate $\left|\phi_{ \pm}(t)\right\rangle$ of $H_{0}(t)$. In other words, the exact state at $t \geq 0$ reads

$$
|\psi(t)\rangle=e^{i f(t)}\left|\phi_{ \pm}(t)\right\rangle
$$

no matter how fast $H_{0}(t)$ varies with time $t$.

By using $\mathbf{b}_{0}(t)$, one finds the explicit form of the TQD field [6]:

$$
\begin{aligned}
\gamma \mathbf{B}(t)= & \left(\omega_{0}-\omega \cos \theta\right) \sin \theta\left[\mathbf{e}_{x} \cos (\omega t)+\mathbf{e}_{y} \sin (\omega t)\right] \\
& +\left(\omega_{0} \cos \theta+\omega \sin ^{2} \theta\right) \mathbf{e}_{z},
\end{aligned}
$$

where from now on the field strength $B_{0}(t)=B_{0}$ is taken to be constant and we have put $\omega_{0}=\gamma B_{0}$. This leads to the TQD Hamiltonian

$$
\begin{aligned}
H(t)= & \frac{1}{2} \hbar\left[\left(\omega_{0}-\omega \cos \theta\right) \sin \theta\right]\left[\cos (\omega t) \sigma_{x}+\sin (\omega t) \sigma_{y}\right] \\
& +\frac{1}{2} \hbar\left(\omega_{0} \cos \theta+\omega \sin ^{2} \theta\right) \sigma_{z} .
\end{aligned}
$$

The refocusing sequence shown in Fig. 1 is

$$
C \rightarrow \pi \rightarrow \bar{C} \rightarrow \pi
$$

where $C$ is the loop traced by the eigenstates $\left|\phi_{ \pm}(t)\right\rangle$ of the Hamiltonian $H_{0}(t)$ exactly driven by $H(t)$, and $\bar{C}(\omega)=$ $C(-\omega)$ is the same loop traced backwards. The $\pi$ transformation is enacted after each loop and described by the Hamiltonian

$$
H(t)=\frac{1}{2} \hbar \omega_{\pi} \sigma_{y},
$$

by applying the magnetic field $\gamma \mathbf{B}_{\pi}(t)=\left(0, \omega_{\pi}, 0\right)$. The sequence can now be described by a Hamiltonian divided up as

$$
H(t)= \begin{cases}H^{(a)}(t), & 0 \leq t \leq \frac{2 \pi}{\omega}, \\ H^{(b)}(t), & t_{1} \leq t \leq t_{1}+\frac{\pi}{\omega_{\pi}}, \\ H^{(c)}(t), & t_{2} \leq t \leq t_{2}+\frac{2 \pi}{\omega}, \\ H^{(d)}(t), & t_{3} \leq t \leq t_{3}+\frac{\pi}{\omega_{\pi}} \equiv \tau,\end{cases}
$$

where $t_{1}>(2 \pi / \omega), t_{2}>t_{1}+\left(\pi / \omega_{\pi}\right)$, and $t_{3}>t_{2}+$ $(2 \pi / \omega)$ (the Hamiltonian is assumed to vanish during the intermediate time intervals $\left[(2 \pi / \omega), t_{1}\right],\left[t_{1}+\right.$ $\left.\left(\pi / \omega_{\pi}\right), t_{2}\right]$, and $\left.\left[t_{2}+(2 \pi / \omega), t_{3}\right]\right)$. Here, the TQD Hamiltonians $H^{(a)}(t)$ and $H^{(c)}(t)$ exactly implement the two spin loops $C$ and $\bar{C}$, respectively, while $H^{(b)}(t)$ and $H^{(d)}(t)$ correspond to the two $\pi$ pulses.

In order for the second loop to retrace the first, the corresponding magnetic fields $\mathbf{B}^{(c)}$ and $\mathbf{B}^{(a)}$ must have different opening angles to the rotational axis. Indeed, from Eq. (4), we find the relative vector $\gamma \Delta \mathbf{B}=\gamma\left(\mathbf{B}^{(a)}-\mathbf{B}^{(c)}\right)=2$ $\sin \theta\left(-\omega \cos \theta \cos \omega t, \omega_{0} \sin \omega t, \omega \sin \theta\right)$, which is clearly nonzero and scales linearly with $\omega$, meaning that the speed of the evolution dictates the difference in applied magnetic fields. This is a direct consequence of the TQD since the $\omega$ factor arose from the correction term, and since the adiabatic regime is entered when $\left|\omega / \omega_{0}\right| \ll 1$, in which the relative vector would be close to zero. The respective magnitudes do not differ because $\gamma\left|\mathbf{B}^{(a)}\right|=\gamma\left|\mathbf{B}^{(c)}\right|=$ $\omega_{0}\left[1+\left[\left(\omega / \omega_{0}\right) \sin \theta\right]^{2}\right]^{1 / 2}$ is unaffected by a sign change of $\omega$. The aspects discussed are illustrated in Fig. 2.

It is worth stressing that the states driven are the eigenstates not of $H(t)$ but of the root Hamiltonian $H_{0}(t)$. The TQD technique enables the evolution to exactly track the instantaneous eigenstates of $H_{0}(t)$, even though the evolution is performed at high speed. A consequence of this is that the Berry phase is unaffected by the TQD, apart from being picked up faster.

The dynamical phases are unaffected too by the TQD. This can be seen by noting that the exact spin states take 


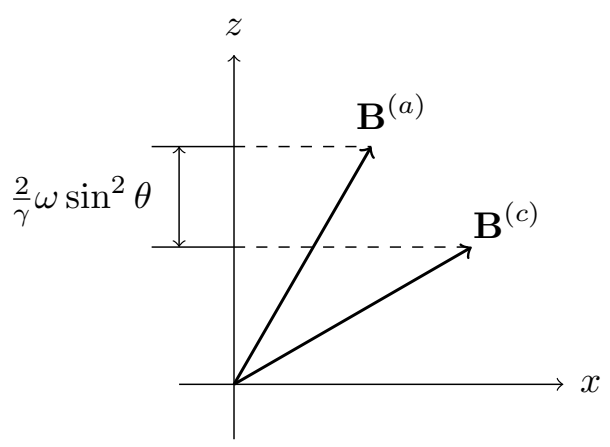

FIG. 2. The difference in magnetic field for the first and second loop, in the $x z$ plane.

the form

$$
\rho_{ \pm}(t)=\frac{1}{2}\left[\hat{1} \pm \mathbf{b}_{0}(t) \cdot \boldsymbol{\sigma}\right]
$$

yielding

$$
\begin{aligned}
& \operatorname{Tr}\left\{\left[\mathbf{b}_{0}(t) \times \partial_{t} \mathbf{b}_{0}(t)\right] \cdot \boldsymbol{\sigma} \rho_{ \pm}(t)\right\} \\
& =\frac{1}{2} \operatorname{Tr}\left\{\left[\mathbf{b}_{0}(t) \times \partial_{t} \mathbf{b}_{0}(t)\right] \cdot \boldsymbol{\sigma}\right\} \\
& \pm \frac{1}{2} \operatorname{Tr}\left\{\left[\mathbf{b}_{0}(t) \times \partial_{t} \mathbf{b}_{0}(t)\right] \cdot \mathbf{b}_{0}(t) \hat{1}\right\}=0
\end{aligned}
$$

where we have used that $\sigma$ is traceless, $\sigma \cdot \sigma=\hat{1}$, and that the triple product $\left[\mathbf{b}_{0}(t) \times \partial_{t} \mathbf{b}_{0}(t)\right] \cdot \mathbf{b}_{0}(t)$ vanishes. In other words, the extra term in the TQD Hamiltonian causes no extra contribution to the spin energy, which implies that the dynamical phases are unaltered.

Apart from the complete suppression of nonadiabatic corrections achieved by TQD, it should be noted that the TQD spin echo scheme can be expected to have a similar kind of resilience to parameter noise and systematic errors to that in standard adiabatic spin echo. The key advantage is the possibility to perform the spin echo sequence at high speed. In this way, the total run time can be made much shorter than that of spin relaxation and dephasing, which, for example, in some QIP implementations may be as short as a few microseconds. This can be achieved by using fields that rotate at frequencies in the order of gigahertz or faster, which is feasible in typical NMR experiments.

\section{APPLICATION: UNIVERSAL GEOMETRIC GATES}

TQD has been used to optimize NMR quantum information processing [11] and to implement geometric quantum gates in nitrogen-vacancy centers [12,13]. Motivated by these earlier results, we demonstrate that spin echo combined with TQD can be used for implementing a set of universal nonadiabatic geometric single- and two-qubit gates, providing means to speed up earlier geometric schemes $[4,5]$ in NMR.

Let us start with the single-qubit case. The root Hamiltonian $H_{0}(t)$ is diagonalized by its instantaneous eigenvectors

$$
\begin{aligned}
& \left|\phi_{0}(t)\right\rangle=\cos \frac{\theta}{2}|0\rangle+\sin \frac{\theta}{2} e^{i \omega t}|1\rangle, \\
& \left|\phi_{1}(t)\right\rangle=-\sin \frac{\theta}{2}|0\rangle+\cos \frac{\theta}{2} e^{i \omega t}|1\rangle
\end{aligned}
$$

with $\sigma_{z}|p\rangle=(1-2 p)|p\rangle, p=0,1$, where for notational convenience we use the conventional qubit notation $|0\rangle,|1\rangle$. Clearly, the initial eigenvectors $\left|\phi_{p}(0)\right\rangle \equiv\left|\phi_{p}\right\rangle$ are parametrized solely by the spherical angle $\theta$, and evolve exactly under the TQD Hamiltonian into the instantaneous eigenvectors $\left|\phi_{p}(t)\right\rangle$ up to phase factors. After completing a loop, each such phase factor comprises a dynamical $\left(\delta_{p}\right)$ and a geometric $\left(\beta_{p}\right)$ component, which, as shown above, are the same as those of adiabatic evolution driven by $H_{0}(t)$ alone. Explicitly, one finds

$$
\begin{aligned}
\delta_{p} & =(1-2 p) \delta, \\
\beta_{p} & =(2 p-1) \frac{1}{2} \Omega,
\end{aligned}
$$

where $\Omega$ is the solid angle enclosed by the loop. Note that while $\delta$ is independent of the orientation of the loop, $\Omega$ changes sign when the loop is reversed. Thus, $\tilde{\delta}_{p}=\delta_{p}$ and $\tilde{\beta}_{p}=-\beta_{p}$, which, applied to the spin echo scheme, results in

$$
\begin{aligned}
\left.\mid \phi_{p}\right) & \stackrel{C_{p}}{\rightarrow} e^{i\left(\delta_{p}+\beta_{p}\right)}\left|\phi_{p}\right\rangle \stackrel{\pi}{\rightarrow} e^{i\left(\delta_{p}+\beta_{p}\right)}\left|\phi_{p \oplus 1}\right\rangle \\
& \stackrel{\bar{C}_{p}}{\rightarrow} e^{i\left(\delta_{p}+\tilde{\delta}_{p \oplus 1}+\beta_{p}+\tilde{\beta}_{p \oplus 1}\right)}\left|\phi_{p \oplus 1}\right\rangle \\
& =e^{i\left(\delta_{p}+\delta_{p \oplus 1}+\beta_{p}-\beta_{p \oplus 1}\right)}\left|\phi_{p \oplus 1}\right\rangle \\
& \stackrel{\pi}{\rightarrow} e^{i\left(\delta_{p}+\delta_{p \oplus 1}+\beta_{p}-\beta_{p \oplus 1}\right)}\left|\phi_{p}\right\rangle
\end{aligned}
$$

where $\oplus$ is addition modulo 2. By combining Eqs. (12) and (13), we see that the spin echo sequence cancels the dynamical phases and thereby results in the purely geometric gate $\left|\phi_{p}\right\rangle \rightarrow U(\Omega)\left|\phi_{p}\right\rangle=e^{i(2 p-1) \Omega}\left|\phi_{p}\right\rangle$, that is,

$$
U(\Omega)=e^{-i \Omega}\left|\phi_{0}\right\rangle\left\langle\phi_{0}\left|+e^{i \Omega}\right| \phi_{1}\right\rangle\left\langle\phi_{1}\right| .
$$

By rewriting this in terms of the computational basis $\{|0\rangle,|1\rangle\}$ via the expressions for the initial eigenstates $\left|\phi_{p}\right\rangle$, one obtains the unitary

$$
U(\theta, \Omega)=e^{-i \Omega \mathbf{n} \cdot \boldsymbol{\sigma}}
$$

where $\mathbf{n}=(\sin \theta, 0, \cos \theta)$.

For $U(\theta, \Omega)$ to be universal, $\theta$ and $\Omega$ must be able to vary independently, but this is clearly not the case since 
$|\Omega|=2 \pi(1-\cos \theta)$, and so another control parameter $\vartheta$ is required. This can be obtained by rotating the TQD magnetic fields around some symmetry axis [14], here, the $y$ axis, such that $\mathbf{B}^{\prime}=R_{y}(\vartheta-\theta) \mathbf{B}$, in turn producing the rotated direction $\mathbf{n}^{\prime}=R_{y}(\vartheta-\theta) \mathbf{n}$, that is,

$$
\begin{aligned}
\mathbf{n}^{\prime} & =\left(\begin{array}{ccc}
\cos (\vartheta-\theta) & 0 & \sin (\vartheta-\theta) \\
0 & 1 & 0 \\
-\sin (\vartheta-\theta) & 0 & \cos (\vartheta-\theta)
\end{array}\right)\left(\begin{array}{c}
\sin \theta \\
0 \\
\cos \theta
\end{array}\right) \\
& =\left(\begin{array}{c}
\sin \vartheta \\
0 \\
\cos \vartheta
\end{array}\right) .
\end{aligned}
$$

The result is the universal unitary

$$
\begin{aligned}
& U(\vartheta, \Omega) \\
& =\left(\begin{array}{cc}
\cos ^{2} \frac{\vartheta}{2} e^{-i \Omega}+\sin ^{2} \frac{\vartheta}{2} e^{i \Omega} & -i \sin \vartheta \sin \Omega \\
-i \sin \vartheta \sin \Omega & \sin ^{2} \frac{\vartheta}{2} e^{-i \Omega}+\cos ^{2} \frac{\vartheta}{2} e^{i \Omega}
\end{array}\right) \\
& =e^{-i \Omega \mathbf{n}^{\prime} \cdot \boldsymbol{\sigma}},
\end{aligned}
$$

where, by definition, $\vartheta$ and $\Omega$ can now be varied independently. Moreover, due to the spherical symmetry the Berry phases remain the same. This is because the same loops are traced, only in a rotated frame, and as such the solid angles remain the same.

For two unitaries $U\left(\vartheta_{1}, \Omega_{1}\right), U\left(\vartheta_{2}, \Omega_{2}\right)$ to contribute to universality it is necessary that $\left[U\left(\vartheta_{1}, \Omega_{1}\right), U\left(\vartheta_{2}, \Omega_{2}\right)\right] \neq$ 0 , which can be shown to equate to [14]

$$
\sin \Omega_{1} \sin \Omega_{2} \sin \left(\vartheta_{1}-\vartheta_{2}\right) \neq 0 .
$$

Simply choosing $\vartheta_{1}-\vartheta_{2} \neq n \pi$, where $n \in \mathbb{Z}$, takes care of the issue. By letting $\vartheta_{1}=0$, the unitary

$$
U\left(0, \Omega_{1}\right)=\left(\begin{array}{cc}
e^{-i \Omega_{1}} & 0 \\
0 & e^{i \Omega_{1}}
\end{array}\right)
$$

is obtained which is equivalent to the $\phi$-gate $|p\rangle \rightarrow$ $e^{i p 2 \Omega_{1}}|p\rangle$ up to a global phase $e^{-i \Omega_{1}}$. Now for the second gate, let $\vartheta_{2}=\pi / 2$; then

$$
U\left(\frac{\pi}{2}, \Omega_{2}\right)=\left(\begin{array}{cc}
\cos \Omega_{2} & -i \sin \Omega_{2} \\
-i \sin \Omega_{2} & \cos \Omega_{2}
\end{array}\right) .
$$

Furthermore, either set $\Omega_{2}=\pi / 2$ to obtain

$$
U\left(\frac{\pi}{2}, \frac{\pi}{2}\right)=\left(\begin{array}{cc}
0 & -i \\
-i & 0
\end{array}\right),
$$

being the equivalent to spin-flip up to the phase factor $-i$, or set $\Omega_{2}=\pi / 4$ to obtain

$$
U\left(\frac{\pi}{2}, \frac{\pi}{4}\right)=\frac{1}{\sqrt{2}}\left(\begin{array}{cc}
1 & -i \\
-i & 1
\end{array}\right),
$$

which is an "equal weighted superposition gate," being roughly equivalent to the Hadamard gate.

We next extend the scheme to enact a geometric twoqubit gate by using the Ising interaction. The original Hamiltonian is

$$
\begin{aligned}
H_{0}(t)= & \gamma_{\mathrm{I}} \mathbf{B}_{0}(t) \cdot \mathbf{S}_{\mathrm{I}} \otimes \hat{1}+\hat{1} \otimes \gamma_{\mathrm{II}} \mathbf{B}_{0}(t) \cdot \mathbf{S}_{\mathrm{II}} \\
& +\frac{2 J}{\hbar} S_{\mathrm{I} ; z} \otimes S_{\mathrm{II} ; z}
\end{aligned}
$$

with I and II denoting the two qubits. By assuming $\gamma_{\mathrm{I}} / \gamma_{\mathrm{II}} \gg 1$, the Hamiltonian reduces to

$$
\begin{aligned}
H_{0}(t)= & \gamma_{\mathrm{I}} \mathbf{B}_{0}^{(0)}(t) \cdot \mathbf{S}_{\mathrm{I}} \otimes|0\rangle\langle 0| \\
& +\gamma_{\mathrm{I}} \mathbf{B}_{0}^{(1)}(t) \cdot \mathbf{S}_{\mathrm{I}} \otimes|1\rangle\langle 1| .
\end{aligned}
$$

We assume the $\mathrm{rf}$ field is swept to resonance, yielding $\gamma_{\mathrm{I}} \mathbf{B}_{0}^{(q)}(t) \equiv \omega_{\mathrm{I}}\left[\cos \omega t, \sin \omega t,(1-2 q) J / \omega_{\mathrm{I}}\right]$ with $q=0,1$ indexing the state of qubit II, and we have defined $\omega_{\mathrm{I}}=$ $\gamma_{\mathrm{I}} B_{0}$. Thus, I plays the role of the target qubit which sees two different effective magnetic fields $\mathbf{B}_{0}^{(q)}(t)$ conditioned on the state of control qubit II. These effective fields are modified in the TQD scheme. If we define

$$
\cos \theta_{q} \equiv(1-2 q) \frac{J}{\sqrt{\omega_{I}^{2}+J^{2}}} \equiv(1-2 q) \cos \tilde{\theta}
$$

the direction of each modified magnetic field is $\mathbf{b}_{0}^{(q)}(t)=$ [ $\cos \omega t \sin \tilde{\theta}, \sin \omega t \sin \tilde{\theta},(1-2 q) \cos \tilde{\theta}$ ]. The correction to the magnetic field in order to achieve transitionless driving is then

$$
\begin{aligned}
\mathbf{b}_{0}^{(q)} \times \partial_{t} \mathbf{b}_{0}^{(q)}= & -(1-2 q) \omega \sin \tilde{\theta} \cos \tilde{\theta} \\
& \times\left(\mathbf{e}_{x} \cos \omega t+\mathbf{e}_{y} \sin \omega t\right) \\
& +\omega \sin ^{2} \tilde{\theta} \mathbf{e}_{z},
\end{aligned}
$$

which defines the full TQD effective fields

$$
\begin{aligned}
\gamma_{\mathrm{I}} \mathbf{B}^{(q)}(t)= & \gamma_{\mathrm{I}} \mathbf{B}_{0}^{(q)}(t)+\mathbf{b}_{0}^{(q)} \times \partial_{t} \mathbf{b}_{0}^{(q)} \\
= & {\left[\omega_{\mathrm{I}}-(1-2 q) \omega \sin \tilde{\theta} \cos \tilde{\theta}\right] } \\
& \times\left(\mathbf{e}_{x} \cos \omega t+\mathbf{e}_{y} \sin \omega t\right) \\
& +\left[(1-2 q) J+\omega \sin ^{2} \tilde{\theta}\right] \mathbf{e}_{z} .
\end{aligned}
$$


The root Hamiltonian in Eq. (24) is diagonalized by its instantaneous eigenvectors

$$
\begin{aligned}
& \left|\phi_{00}(t)\right\rangle=\cos \frac{\tilde{\theta}}{2}|00\rangle+e^{i \omega t} \sin \frac{\tilde{\theta}}{2}|10\rangle, \\
& \left|\phi_{10}(t)\right\rangle=-\sin \frac{\tilde{\theta}}{2}|00\rangle+e^{i \omega t} \cos \frac{\tilde{\theta}}{2}|10\rangle, \\
& \left|\phi_{01}(t)\right\rangle=\sin \frac{\tilde{\theta}}{2}|01\rangle+e^{i \omega t} \cos \frac{\tilde{\theta}}{2}|11\rangle, \\
& \left|\phi_{11}(t)\right\rangle=-\cos \frac{\tilde{\theta}}{2}|01\rangle+e^{i \omega t} \sin \frac{\tilde{\theta}}{2}|11\rangle,
\end{aligned}
$$

where we have used that $\theta_{0}=\tilde{\theta}$ and $\theta_{1}=\pi-\tilde{\theta}$. The refocusing scheme extended to two qubits is

$$
C \rightarrow \pi_{\mathrm{I}} \rightarrow \bar{C} \rightarrow \pi_{\mathrm{II}} \rightarrow C \rightarrow \pi_{\mathrm{I}} \rightarrow \bar{C} \rightarrow \pi_{\mathrm{II}},
$$

where the Berry phases picked up are $\beta_{p q}=(2 p-1) \frac{1}{2} \Omega_{q}$ with

$$
\begin{aligned}
\left|\Omega_{q}\right| & =2 \pi[1-(1-2 q) \cos \tilde{\theta}] \\
& =2 \pi\left[1-(1-2 q) \frac{J}{\sqrt{\omega_{\mathrm{I}}^{2}+J^{2}}}\right] .
\end{aligned}
$$

Again, the solid angle changes sign depending on the direction of the path, thus $\tilde{\beta}_{p q}=-\beta_{p q}$, while $\tilde{\delta}_{p q}=\delta_{p q}$. The spin echo scheme results in

$$
\left|\phi_{p q}\right\rangle \rightarrow e^{i\left(\beta_{p q}-\beta_{p \oplus 1, q}+\beta_{p \oplus 1, q \oplus 1}-\beta_{p, q \oplus 1}\right)}\left|\phi_{p q}\right\rangle .
$$

Thus, the refocusing scheme enacts a purely geometric gate $\left|\phi_{p q}\right\rangle \rightarrow U(\Delta \Omega)\left|\phi_{p q}\right\rangle=e^{(-1)^{p+q} 2 i \Delta \Omega}\left|\phi_{p q}\right\rangle$, that is,

$$
\begin{aligned}
U= & e^{2 i \Delta \Omega}\left(\left|\phi_{00}\right\rangle\left\langle\phi_{00}|+| \phi_{11}\right\rangle\left\langle\phi_{11}\right|\right) \\
& +e^{-2 i \Delta \Omega}\left(\left|\phi_{01}\right\rangle\left\langle\phi_{01}|+| \phi_{10}\right\rangle\left\langle\phi_{10}\right|\right),
\end{aligned}
$$

where we have defined the differential solid angle $\Delta \Omega \equiv$ $\left(\Omega_{1}-\Omega_{0}\right) / 2$. Again, the prerequisite rotation of the magnetic field is required so that the substitution $(\pi / 2) \rightarrow \vartheta$ can be made. This makes the unitary in the computational basis parameter dependent as $U\left(\vartheta_{0}, \vartheta_{1}, \Delta \Omega\right)$. By choosing $\vartheta_{0}=\vartheta_{1}=0$, the phase gate

$$
\begin{aligned}
U(0,0, \Delta \Omega)= & e^{2 i \Delta \Omega}(|00\rangle\langle 00|+| 11\rangle\langle 11|) \\
& +e^{-2 i \Delta \Omega}(|01\rangle\langle 01|+| 10\rangle\langle 10|)
\end{aligned}
$$

is obtained. This is a conditional gate where a phase difference of $4 \Delta \Omega$ is picked up depending on whether the qubits are parallel or not.
Since the TQD correction $\mathbf{b}_{0}^{(q)} \times \partial_{t} \mathbf{b}_{0}^{(q)}$ of the effective magnetic field $\mathbf{B}_{q}(t)$ seen by target qubit I is conditionalized upon the state of control qubit II, it becomes difficult to realize experimentally the TQD Hamiltonian directly. This can be resolved by reformulating the system in terms of the time independent Hamiltonian

$$
\begin{gathered}
H_{\exp }=\omega_{\mathrm{I}}^{\prime} \sin \theta^{\prime} S_{x} \otimes \hat{1}+\omega_{\mathrm{I}}^{\prime} \cos \theta^{\prime} S_{z} \otimes \hat{1} \\
+\frac{2 J_{z z}}{\hbar} S_{z} \otimes S_{z}+\frac{2 J_{x z}}{\hbar} S_{x} \otimes S_{z}
\end{gathered}
$$

as the starting point for the scheme. In this way, the TQD Hamiltonian can be simulated by rotating the sample described by $H_{\exp }$ with angular frequency $\omega$ around the $z$ axis. This can be seen by evaluating the Hamiltonian in the rotating frame, yielding

$$
\begin{aligned}
\tilde{H}_{\exp }(t)= & \gamma_{1} \tilde{\mathbf{B}}_{\exp }^{(0)}(t) \cdot \mathbf{S} \otimes|0\rangle\langle 0| \\
& +\gamma_{\mathrm{I}} \tilde{\mathbf{B}}_{\exp }^{(1)}(t) \cdot \mathbf{S} \otimes|1\rangle\langle 1|+\omega \hat{1} \otimes S_{z}
\end{aligned}
$$

with

$$
\begin{aligned}
\gamma_{\mathrm{I}} \tilde{\mathbf{B}}_{\exp }^{(q)}= & {\left[\omega_{\mathrm{I}}^{\prime} \sin \theta^{\prime}+(1-2 q) J_{x z}\right]\left(\mathbf{e}_{x} \cos \omega t+\mathbf{e}_{y} \sin \omega t\right) } \\
& +\left[\omega_{\mathrm{I}}^{\prime} \cos \theta^{\prime}+\omega+(1-2 q) J_{z z}\right] \mathbf{e}_{z} .
\end{aligned}
$$

Now, the extra control qubit term $\omega \hat{1} \otimes S_{z}$ in Eq. (35) commutes with the Hamiltonian $\tilde{H}_{\text {exp }}(t)$ and will therefore be canceled by the spin echo. We may thus simulate the effect of the two-qubit TQD system by a suitable choice of experimental parameters so that Eqs. (36) and (27) coincide. This yields

$$
\begin{aligned}
J_{x z} & =-\omega \sin \tilde{\theta} \cos \tilde{\theta}=-\frac{\omega \omega_{\mathrm{I}}}{\omega_{\mathrm{I}}^{2}+J^{2}} J, \\
J_{z z} & =J, \\
\tan \theta^{\prime} & =-\frac{\omega_{\mathrm{I}}}{\omega \cos ^{2} \tilde{\theta}}=-\frac{\omega_{\mathrm{I}}}{\omega J^{2}}\left(\omega_{\mathrm{I}}^{2}+J^{2}\right), \\
\omega_{\mathrm{I}}^{\prime} & =\sqrt{\omega_{\mathrm{I}}^{2}+\omega^{2} \cos ^{4} \tilde{\theta}} \\
& =\frac{\sqrt{\omega_{\mathrm{I}}^{2}\left(\omega_{\mathrm{I}}^{2}+J^{2}\right)^{2}+\omega^{2} J^{4}}}{\omega_{\mathrm{I}}^{2}+J^{2}} .
\end{aligned}
$$

Other high-speed geometric gates have been developed [14-17] in the past. These gates share with our proposed one- and two-qubit gates that they are all based on the exact unitary evolution of quantum states. On the other hand, while these earlier proposals use nonadiabatic geometric phase concepts, such as the Aharonov and Anandan [18] and Manini and Pistolesi [19] phases, the geometric nature of our gates follows from its explicit relation to the adiabatic Berry phase of the root Hamiltonian $H_{0}(t)$. 
In this way, our scheme combines the high-speed nature of nonadiabatic geometric quantum computation [14-17] with parametric control of its adiabatic counterpart [4,5].

\section{CONCLUSIONS}

A technique for accurate spin control based on a combination of spin echo and transitionless quantum driving has been proposed. This provides a means to perform all elements in the spin echo sequence at high speed. We have demonstrated that the technique can be used to implement robust one- and two-qubit geometric gates in NMR quantum information processing. This form of high-speed geometric gates differs from previous proposals of nonadiabatic geometric quantum computation. Another potentially important technological application of the highspeed spin echo scheme is MRI, for which the suppression of nonadiabatic transitions may help to improve scanning resolution.

\section{ACKNOWLEDGMENTS}

E.S. acknowledges support from Swedish Research Council (VR) Grant No. 2017-03832.

[1] E. L. Hahn, Spin echoes, Phys. Rev. 80, 580 (1950).

[2] H. Y. Carr and E. M. Purcell, Effects of diffusion on free precession in nuclear magnetic resonance experiments, Phys. Rev. 94, 630 (1954).

[3] M. V. Berry, Quantal phase factors accompanying adiabatic changes, Proc. R. Soc. London, Ser. A 392, 45 (1984).

[4] J. A. Jones, V. Vedral, A. Ekert, and G. Castagnoli, Geometric quantum computation using nuclear magnetic resonance, Nature 403, 869 (2000).

[5] A. Ekert, M. Ericsson, P. Hayden, H. Inamori, J. A. Jones, D. K. L. Oi, and V. Vedral, Geometric quantum computation, J. Mod. Opt. 47, 2501 (2000).

[6] M. V. Berry, Transitionless quantum driving, J. Phys. A: Math. Theor. 42, 365303 (2009).
[7] R. W. Darbeau, Nuclear magnetic resonance (NMR) spectroscopy: A review and a look at its use as a probative tool in deamination chemistry, Appl. Spectrosc. Rev. 41, 401 (2006).

[8] B. A. Jung and M. Weigel, Spin echo magnetic resonance imaging, J. Magn. Reson. Imaging 37, 805 (2013).

[9] L. M. K. Vandersypen and I. L. Chuang, NMR techniques for quantum control and computation, Rev. Mod. Phys. 76, 1037 (2005).

[10] Z. Zhang, T. Wang, L. Xiang, J. Yao, J. Wu, and Y. Yin, Measuring the berry phase in a superconducting phase qubit by a shortcut to adiabaticity, Phys. Rev. A 95, 042345 (2017).

[11] A. C. Santos, A. Nicotina, A. M. Souza, R. S. Sarthour, I. S. Oliveira, and M. S. Sarandy, Optimizing NMR quantum information processing via generalized transitionless quantum driving, Europhys. Lett. 129, 30008 (2020).

[12] Z.-T. Liang, X. Yue, Q. Lv, Y.-X. Du, W. Huang, H. Yan, and S.-L. Zhu, Proposal for implementing universal superadiabatic geometric quantum gates in nitrogen-vacancy centers, Phys. Rev. A 93, 040305(R) (2016).

[13] F. Kleißler, A. Lazariev, and S. Arroyo-Camejo, Universal, high-fidelity quantum gates based on superadiabatic, geometric phases on a solid-state spin-qubit at room temperature, Npj Quantum Inf. 4, 49 (2018).

[14] S.-L. Zhu and Z. D. Wang, Implementation of Universal Quantum Gates Based on Nonadiabatic Geometric Phases, Phys. Rev. Lett. 89, 097902 (2002).

[15] W. Xiang-Bin and M. Keiji, Nonadiabatic Conditional Geometric Phase Shift with NMR, Phys. Rev. Lett. 87, 097901 (2001).

[16] L. B. Shao, Z. D. Wang, and D. Y. Xing, Implementation of quantum gates based on geometric phases accumulated in the eigenstates of periodic invariant operators, Phys. Rev. A 75, 014301 (2007).

[17] M. Ericsson, D. Kult, E. Sjöqvist, and J. Åberg, Nodal free geometric phases: Concept and application to geometric quantum computation, Phys. Lett. A 372, 596 (2008).

[18] Y. Aharonov and J. Anandan, Phase Change during a Cyclic Quantum Evolution, Phys. Rev. Lett. 58, 1593 (1987).

[19] N. Manini and F. Pistolesi, Off-Diagonal Geometric Phases, Phys. Rev. Lett. 85, 3067 (2000). 